\title{
Aberrant patterns of brain cerebral blood flow in Chinese han first-episode drug-naïve depressive patients with and without a family history of depression
}

\author{
Shikai Wang ${ }^{1, *}$, Lina Wang ${ }^{2, *}$, Ping Jing ${ }^{3, *}$, Ping Guo ${ }^{1}$, Weifang $\mathrm{Zheng}^{3}$, Jie $\mathrm{Li}^{2}$ and \\ Mincai Qian ${ }^{1}$ \\ ${ }^{1}$ Department of Psychological Medicine, Huzhou Third People's Hospital, Huzhou, China \\ ${ }^{2}$ Department of Psychological Medicine, Tianjin Anding Hospital, Tianjin, China \\ ${ }^{3}$ Department of Psychological Medicine, Wenzhou Seventh People's Hospital, Wenzhou, China \\ *These authors contributed equally to this work
}

Correspondence to: Mincai Qian, email: mincaiqian@163.com

Jie Li, email: tjlijie3827@163.com

Keywords: depression, family history, pCASL, regional cerebral blood flow, aberrant pattern

Received: June 08, 2017 Accepted: August 04, $2017 \quad$ Published: August 17, 2017

Copyright: Wang et al. This is an open-access article distributed under the terms of the Creative Commons Attribution License 3.0 (CC BY 3.0), which permits unrestricted use, distribution, and reproduction in any medium, provided the original author and source are credited.

\section{ABSTRACT}

A positive family history plays a key role in the brain pathology of depression patients and previous research has confirmed that disturbed mood maintenance may be related to abnormal regional cerebral blood flow (rCBF). However, little is known about whether the rCBF is different between depression patients with and without family histories. To address this question, we examined the rCBF in drug-naïve, firstepisode depression patients with and without family histories of depression using a 3D pseudo-continuous arterial spin-labelling technique. We found that decreased rCBF was predominantly observed in the patients without family histories, while decreased and increased rCBF co-existed in patients with family histories. The observed brain regions with altered $\mathrm{rCBF}$ were associated with affection processing, such as the prefrontal, occipital and insular areas. However the patterns of rCBF alteration observed in the present study were different from those found in previous studies where patients were compared with healthy controls. Our present findings, together with the findings from previous studies have prompted the need of a long-term follow-up study to characterize the brain features of the developmental trajectory of depression and investigate the targets for precise, personalized treatments.

\section{INTRODUCTION}

Many previous studies confirmed that genetics play a key role in the etiology of major depressive disorder $[1,2]$ and many previous studies confirmed that depression patients with a positive family history are more inclined to develop treatment refractory depression [3]. Depression in patients with a positive family history is usually considered to be mainly associated with genetic factors, but depression in patients with a negative family history is usually attributed to environmental factors [4]. More notably, some studies have reported that differences can be observed in the expression of depressive symptoms, cognitive impairments, and treatment outcomes between depression patients with and without family histories $[5,6]$.

Several previous neuroimaging studies based on Magnetic Resonance Imaging (MRI) have focused on structural and functional alterations between depression patients and healthy controls. For example, one previous study found that some brain regions located in the prefrontal area and the limbic system demonstrated structural aberrations and that the connectivity between these brain regions was also affected, including the ventromedial prefrontal cortex, the dorsolateral prefrontal cortex, the anterior cingulate cortex, the lateral orbital prefrontal cortex, the amygdala, the hippocampus and 
the ventral striatum [7]. At the same time, some studies reported that the functional connectivity between the amygdala and the anterior cingulate cortex regions is also unusual in depression patients compared to healthy controls [8]. Furthermore, a previous study found that the regional blood flow abnormally increased in the ventromedial prefrontal cortex and the lateral orbital prefrontal cortex, but decreased in the dorsolateral prefrontal cortex in depression patients [9]. Additionally, many studies have been confirmed that local spontaneous neural activity is abnormal in depression patients, including amplitude of low frequency fluctuation (ALFF), regional homogeneity (Reho), functional connectivity density (FCD), etc. [10-14]. For example, previous study found that decreased ReHo were located in the left insula, superior temporal gyrus, inferior frontal gyrus, lingual gyrus and cerebellumanterior lobe in the patients with treatment resistant depression (TRD), simultaneously, increased ReHo were found in the left superior temporal gyrus, cerebellum posterior lobe, cerebellum anterior lobe, the right cerebellar tonsil and bilateral fusiform gyrus in the TRD patients [15]. This study supported the hypothesis that the abnormality hypothesis of limbic-cortical networks in major depressive disorder (MDD) [16]. Liu et al. found that patients with MDD exhibited significantly decreased fractional amplitude of low frequency fluctuation (fALFF) in right cerebellum posterior lobe, left parahippocampal gyrus and right middle frontal gyrus and increased fALFF in left superior occipital gyrus/cuneus [17]. Guo et al. found that exhibited reduced fractional amplitude of lowfrequency fluctuations in the right middle frontal gyrus (orbital part) and decreased network homogeneity in the left middle frontal gyrus [18, 19]. Zhuo et. found that decreased global FCD was located in the left postcentral and precentral gyri, right fusiform gyrus and lingual gyrus [20]. Taken together, these findings inclined to support the hypothesis that decreased regional activity and network homogeneity in the frontal cortex may be the key impairment of the fronto-limbic network in the patients with MDD [19]. Collectively, all of the abovementioned findings converge to suggest that the brain regions that participate in emotion regulation, reward regulation, memory and executive function regulation all show structural and functional abnormalities and cerebral blood follow disturbances.

Certain studies have found that cerebral perfusion alterations are predominantly located in the anterior cingulate cortex, right medial prefrontal cortex, temporal cortex, hippocampus, thalamus and cerebellum [21, 22]. Vasic found that $\mathrm{CBF}$ was reduced in the anterior cingulate and bilateral parahippocampal areas and increased in the frontoparietal and striatal regions in MDD patients [23]. Drevets summarized that the brain $\mathrm{rCBF}$ in patients with depression showed a general tendency to decrease and that the brain regions with abnormal $\mathrm{CBF}$ in depression patients predominantly included the frontal lobe, temporal lobe, cingulate cortex, amygdala, caudate nucleus, and paralimbic system [14]. All the aforementioned studies converged to suggest that the alterations of $\mathrm{CBF}$ in the regions located in the limbic-subcortical-cortical circuit appear to be common brain characteristics of patients with depression [24]. However, although previous findings related to $\mathrm{rCBF}$ enhanced our understanding of the pathological features of major depressive disorder, few studies have reported the difference between depression patients with and without family histories.

The pCASL technique is a recently developed method used to acquire information on cerebral blood flow. In the last five years, many studies adopted pCASL to study mental disorders and discovered many important findings $[22,25]$. Based on previous studies, in the current study, we tried to investigate the cerebral blood flow differences in drug-naïve, first-episode depression patients with and without family histories using the 3D pCASL technique [26]. We hypothesized that the aberrant pattern of $\mathrm{rCBF}$ is different between these two groups, the $\mathrm{rCBF}$ alterations in the MDD patients with a family history will more widely and complexity than the MDD patients without a family history.

\section{RESULTS}

\section{Comparison of the brain regions with altered cerebral perfusion between depression patients with a family history of depression and the healthy control group}

Compared to the healthy controls, depression patients with a family history showed significantly increased $\mathrm{CBF}$ in the bilateral basal ganglia, the bilateral middle temporal gyrus, the lateral inferior gyrus, the left paracentral gyrus, and the right thalamus. Significantly decreased $\mathrm{CBF}$ was observed in the bilateral prefrontal lobe, the insular, the occipital lobe and the right medial temporal lobe (Figure 1).

\section{Comparison of the brain regions with altered cerebral perfusion between depression patients without a family history of depression and the healthy control group}

Compared to the healthy controls, unlike the depression patients with a family history, significantly decreased $\mathrm{rCBF}$ was only observed in the bilateral insular, the left prefrontal lobe and the right occipital lobe. No significantly increased CBF brain regions were observed in these patients without a family history (Figure 2).

\section{Relationship between rcbf alterations and hamd scores}

We calculated the correlations between the $\mathrm{rCBF}$ alterations and HAMD scores in the patients with and 
without family histories. Unfortunately, we did not find any correlations.

\section{DISCUSSION}

In current study, we first adopted pCASL methods to explore $\mathrm{rCBF}$ abnormalities in first-episode, drug-naïve depression patients with and without family histories of depression. We found distinct $\mathrm{rCBF}$ alterations between the depression patients with and without family histories compared to the healthy controls. The most important finding in our present study is that we found decreased $\mathrm{rCBF}$ only in the depression patients without a family history compared to the healthy controls. However, decreased and increased $\mathrm{rCBF}$ co-existed in the patients with a family history. The distribution of rCBF alterations in the depression patients with a family history is more widespread than in the patients without a family history. In addition, increased $\mathrm{rCBF}$ is also evident in the patients with a family history. In summary, our findings suggest that the depression patients with a family history had more extensive cerebral blood disturbance than the patients without a family history.

A strength of our study is that we enrolled drugnaïve, first-episode patients. Therefore, our findings were unlikely to be influenced by confounding factors (such as previous therapy). This allows our findings to more objectively reflect the pathological features of these patients. The common $\mathrm{rCBF}$ alteration in the two patient groups was the decreased $\mathrm{rCBF}$ in the prefrontal

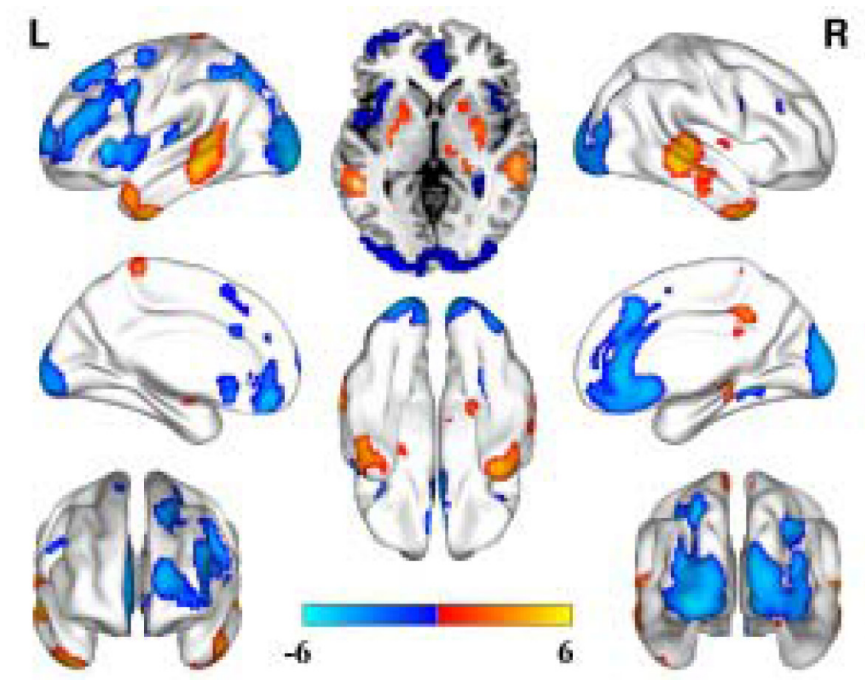

Figure 1: CBF alterations in the depression patients with a family history compared to the healthy controls (FDR correction, $P<\mathbf{0 . 0 5}$, cluster size $\geq \mathbf{5 0}$ ).

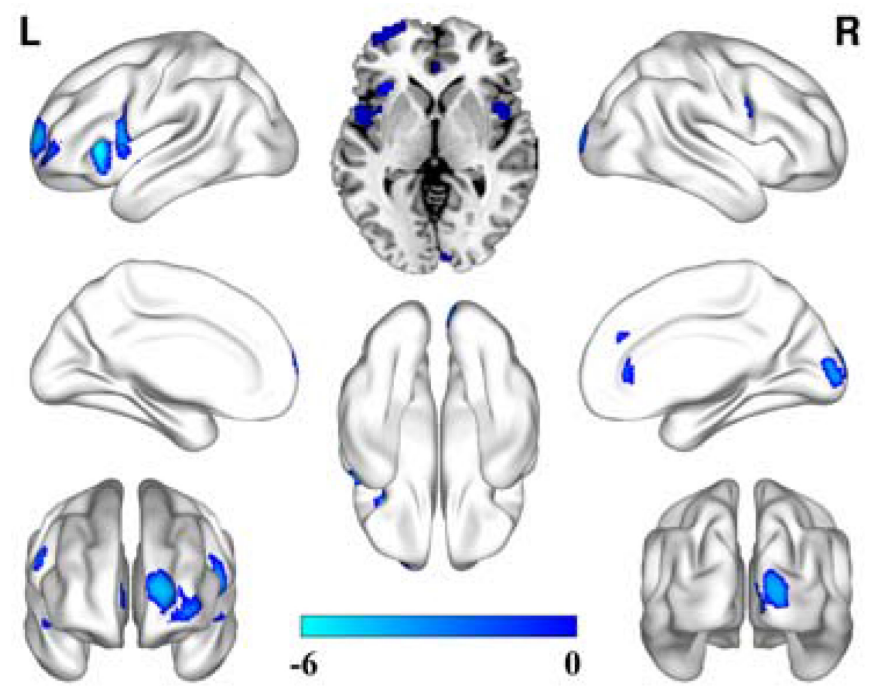

Figure 2: CBF alterations in the depression patients without a family history compared to the healthy controls (FDR correction, $P<\mathbf{0 . 0 5}$, cluster size $\geq \mathbf{5 0}$ ). 
lobe, the insular and the occipital lobe areas. The specific alteration in the patients with a family history was the increased $\mathrm{rCBF}$ in the bilateral basal ganglia, the bilateral middle temporal gyrus, the lateral inferior gyrus, the left paracentral gyrus, the right thalamus, these findings supported the hypothesis that increased cerebellar-defaultmode-network connectivity in MDD patients to some extent [27]. Previous studies confirmed that functional or structural alterations in the prefrontal-limbic system participated in the regulation of affection, the regulation of memory processes and executive function [7, 28-31]. Previous studies have also reported that decreased $\mathrm{rCBF}$ was also found in the prefrontal cortex and the orbital prefrontal cortex and that these brain regions participated in the regulation of aggression, sexual functioning and perseverative affection processing [23, 31-33]. In addition, decreased $\mathrm{rCBF}$ in the insular in our present study supported the hypothesis that insular act as an integration center of emotional processing which is disrupted in the depressed patients [34]. Most of our current findings are consistent with the previous studies and together support the hypo-metabolism hypothesis of depression.

However, some inconsistencies were also observed between our current study and previous studies. For example, we found increased $\mathrm{rCBF}$ in the bilateral basal ganglia, the bilateral middle temporal gyrus, the lateral inferior gyrus, the left paracentral gyrus and the right thalamus. This finding did not completely support the previous hypothesis to some extent [7, 27-34]. However, this is not entirely incomprehensible. About the $\mathrm{rCBF}$ alterations in MDD patients, inconsistent findings reported by different studies, to the best of our knowledge, there is none a completely consistent findings was reported by two studies. Just as Drevets summarized and reported by the meta-analysis of Gong, the brain $\mathrm{rCBF}$ in MDD patients demonstrated a general decrease tendency which predominantly located in the limbic-subcortical-cortical circuit $[14,24]$. Simultaneously, in these studies included in the meta-analysis and review, also some studies reported increased $\mathrm{rCBF}$ in some brain regions and many inconsistent findings among these studies [14, 27]. We cannot completely explain this difference based on our current knowledge. The probably reasons we can suggest are caused by many influence factors' differences in different studies, such as patient differences, MRI parameters, antidepressants, et al. $[35,36]$. The inconsistent findings provide pivotal information for us to consider that we should conduct a long-term follow-up study which adopt a uniform enrolled criteria and uniform MRI parameters to test this and to explore the dynamic alterations in drug naive depression patients from the first episode to acquired appropriate treatments. Then, we can characterize the brain features of the developmental trajectory of depression and investigate the treatment targets for precise, personalized treatments.

A unique phenomenon was observed in our study. When we compared the two patient groups, we found that patients with a family history exhibited increased rCBF in the bilateral middle temporal and the hippocampus brain regions, but this difference cannot withstand the FDR correction. This observation also warrants further investigation. The reasons for the lack of differences between the patients with and without a family histories in the current study can be explained as follows. First, genetic influences on $\mathrm{rCBF}$ alterations have rarely been reported in previous studies, warranting further investigations. Second, as previous studies reported that $\mathrm{rCBF}$ was influenced by many factors such as age, gender, illness duration, MRI parameters, et al. [35], this may account for our current findings. Third, our present study had a flawed design. We need a well-designed study to further explore the differences between these groups so that we can provide more useful information to understand the pathological mechanisms of MDD patients with and without family histories and propose potential mechanisms underlying the influence of genetic factors on $\mathrm{rCBF}$.

\section{Limitation}

Cardiac noise, respiratory noise, low frequency fluctuations and artefacts from physiological noise are especially prevalent in the frequency bands examined at rest. Therefore, Murphy $\mathrm{K}$ et al. advised a pre-processing step to remove physiological noise from the data using simultaneously collected pulse and respiration data [35]. From this perspective, we must collect physiological data in the study. This is a limitation of our present study, and we must consider these factors in the future study. More interestingly, previous studies found that pulse and respiration can influence the $\mathrm{CBF}$ measurement in some special conditions. For example, using volunteers, Kolbitsch reported that continuous positive airway pressure (CPAP) (12 cm H2O) can increase cerebral perfusion [36]. However, Zelaya confirmed that no correlation was found between regional $\mathrm{CBF}$ changes and respiratory rate or heart rate [37]. Therefore, the effects of pulse and respiration on CBF are inconsistent in different studies, thus warranting further study for clarification. In the present study, we did not collect pulse and respiration data in the MDD patients and healthy controls. This decision may represent a limitation to some extent, but these factors cannot completely elicit the physiological noise influence. We must conduct future research according to Murphy K's suggestion. Eklund et al. proposed that a voxel defining threshold of 0.001 is probably a reasonable control under current RFT options, though non-parametric tests would be preferable [38]. In current study, when we adopted this method, all the significant differences were disappeared, this is of great concern, in the future study, we must adopt the Eklund et al. proposed methods to analysis the data to acquire more accurately findings. 


\section{MATERIALS AND METHODS}

\section{Subjects}

All of the patients were diagnosed by two senior professional psychiatrists who adopted the SCI-D according to the criteria for major depressive disorder at the first episode in the DSM-IV (TR Version). An additional criterion was that the patients had not accepted any regular therapeutic or physical treatments in the last two weeks. Another additional criterion was that the family history was also diagnosed by two senior professional psychiatrists according to guardian's reports and medical records. All of the healthy controls were excluded by two professional psychiatrists who adopted the SCI-D (NP Version). The 17-item version Hamilton Rating Scale for Depression (HAMD) [39] was used to assess the severity of the depressive symptoms in the patients. A total of 83 patients were enrolled from January 2015 to December 2016 in the current study. The mean age of the patients with a positive family was $33.6 \pm 7.9$ years and the mean illness duration was $130.8 \pm 93.8$ (days). The mean age of the patients with a negative family history was $34.7 \pm 6.5$ years and the mean illness duration was $116.2 \pm 95.4$ (days). A total of 47 healthy controls were recruited from the hospital staff of Huzhou 3rd Hospital (Huzhou, Zhejiang) and Wenzhou seventh people's hospital (Wenzhou, Zhejiang), with a mean age of $33.0 \pm 7.7$ years. Two senior psychiatrists ruled out a diagnosis of potential mental disorder and the volunteers were only enrolled if they did not have a positive family history of mental disorder. No significant differences were observed among the three groups with regard to age or gender (Table 1). The common exclusion criteria were as follows: participants with a history of unconsciousness for $\geq 5$ min; a neurological disease diagnosis history; a severe mental disorder such as schizophrenia; drug abuse; serious physical illness; patients who were pregnant or lactating; participation in any other research study; treatment within the last two weeks; or endocrine disease and other contraindication for MRI scanning. The Ethics Committee of Hu Zhou 3rd Hospital and Wenzhou Seventh people's Hospital all approved the current study. All participants fully understood the current study purposes and provided written informed consent.

\section{Methods}

\section{MRI image}

We used the Philips Achieva 3.0 T MRI system (Philips Medical Systems Nederland B.V., The Netherlands) to perform the scans. The participants were arranged in a comfortable position and were given special earplugs to minimize the impact of noise during the scanning. A foam pad was placed around each participant's head to control head movement. The scope of MRI scanning included the whole brain. The pCASL scanning parameters were as follows: Echo time/repetition time, 4,000 ms/14 ms; slice thickness, $7 \mathrm{~mm}$; slice number, 17; matrix $=80 \times 80$; interlayer spacing, $1 \mathrm{~mm}$; voxel size, $3 \times 3 \times 7 \mathrm{~mm}$; label spacing, $20 \mathrm{~mm}$; labeling duration, $1,650 \mathrm{~ms}$; label delay, 1,525 ms; and FOV (field of view), $240 \times 240 \mathrm{~mm}$.

\section{Functional MRI data preprocessing}

In the present study, common data processing software for brain functions were used, including Statistics Parameter Mapping (SPM8; Wellcome Department of Imaging Neuroscience, London, UK), and the Relative Expression Software Tool was used to conduct the data preprocessing. The specific process consisted of the following steps. First, for data format conversion, the ASL images from the scans were processed with the built-in software for digital subtraction to obtain the perfusion map and the format was then converted to a dual file format for SPM processing. This step was completed with MRICroN software 1.40 (http://www.cabiatl. com/mricro/ mricro/mricro.html). Second, for spatial normalization, the individual perfusion image was input into a standard PET template provided by SPM8. Third, for the removal of non-brain tissues, the normalized image was computed with a standard mask to remove non-brain tissue. This process was performed using the ImCalc function of SPM8. Fourth, for Gaussian smoothing, a Gaussian kernel of $4 \times 4 \times 4 \mathrm{~mm} 3$ full width at half maximum (FWHM) was used to perform the spatial smoothing of the images, reduce the remaining inter-individual differences after normalization, and improve the SNR. Fifth, mean whole brain perfusion was extracted to obtain a normalized image for each individual. The inter-individual differences in brain perfusion were removed using the individual whole brain perfusion information/average individual whole brain perfusion information. This was performed by the REST and the ImCalc functions of SPM8. An index of transient head motion (AFNI's@1dDiffMag) was calculated from each subject's motion parameters for use as a nuisance covariate in the group-level analyses. This was performed by the REST and the ImCalc functions of SPM8.

\section{Statistical analyses}

SPM8 was used to conduct the statistical analyses of the brain perfusion data between the two groups and to demonstrate the final results. An independent one-sample $t$-test was conducted for the healthy control and patient groups. Age and gender were considered as the covariates to generate the difference map of cerebral blood perfusion between any two groups. The results were put into a single T1 template using the xjView software. Difference comparisons were corrected by the false discovery rate (FDR) method with a significance threshold of $P<0.05$. 
Table 1: Demographic and clinical characteristics of the participants

\begin{tabular}{lcccc}
\hline & $\begin{array}{c}\text { FDD+PF } \\
(\boldsymbol{n}=\mathbf{4 7})\end{array}$ & $\begin{array}{c}\text { FDD+NF } \\
(\boldsymbol{n}=\mathbf{3 6})\end{array}$ & $\begin{array}{c}\text { HC } \\
(\boldsymbol{n}=\mathbf{4 7})\end{array}$ & $33.0 \pm 7.7$ \\
\hline Age (yrs) & $33.6 \pm 7.9$ & $34.7 \pm 6.5$ & $27 / 20$ & 0.600 \\
Sex (M/F) & $28 / 19$ & $20 / 16$ & 0.934 \\
Illness duration, month & $130.8 \pm 93.8$ (days) & $116.2 \pm 95.4$ (days) & $<0.488$ \\
HRSD score & $37.21 \pm 11.24$ & $27.78 \pm 7.64$ & $<01$ \\
\hline
\end{tabular}

FDD+PF: First-episode, drug-naïve depression patients with a positive family history; FDD+NF: First-episode, drug-naïve depression patients with a negative family history; HC: healthy controls; HRSD: 17-item version Hamilton Rating Scale for Depression.

SPSS 19.0 statistical analysis software (SPSS, Inc., Chicago, IL, USA) was adopted for all statistical analyses of all data from the two groups. The statistical analyses were performed using two independent sample $t$-tests and $P<0.05$ was considered to indicate a statistically significant difference. The data are presented as the mean \pm standard deviation.

\section{CONCLUSIONS}

In conclusion, we found that compared to healthy controls, the rCBF alteration patterns in drug-naïve firstepisode depression patients with and without family histories were different. Decreased cerebral perfusion was predominantly observed in the patients without family histories, but decreased and increased rCBF coexisted in the patients with family histories. Compared to healthy controls, the MDD patients with and without family histories all showed decreased $\mathrm{rCBF}$ in the prefrontal lobe, the insular, and the occipital lobe. These altered $\mathrm{rCBF}$ brain regions were related to the brain regions that are associated with affection processing. The more important finding in our study is that there are no differences between MDD patients with and without family histories of depression after FDR correction. These critical counterintuitive findings in the present study have prompted us to conduct a long-term followup study and avoid confounding factors in first-episode, drug-naive depression patients to acquire appropriate treatments. Therefore, we can characterize the brain features of the developmental trajectory of depression and investigate the treatment targets for precise, personalized treatments.

\section{ACKNOWLEDGMENTS}

The authors thank all the research staff for their team collaboration work and all the patients and oncologists involved in this study for their cooperation and support.

\section{CONFLICTS OF INTEREST}

The authors declare no conflicts of interest.

\section{GRANT SUPPORT}

The present study was supported by grants from the Science and technology fund of Hu Zhou (grant no. 2014GZ12 to Mincai Qian). Key Project of Tianjin Health Association (13KG118 to J.L.).

\section{REFERENCES}

1. Galecki P, Talarowska M. Neurodevelopmental theory of depression. Prog Neuropsychopharmacol Biol Psychiatry. 2017 May 19. [Epub ahead of print].

2. Saveanu RV, Nemeroff CB. Etiology of depression: genetic and environmental factors. Psychiatr Clin North Am. 2012; 35:51-71.

3. Burcusa SL, Iacono WG. Risk for recurrence in depression. Clin Psychol Rev. 2007; 27:959-985.

4. Hogenelst K, Schoevers RA, Aan Het Rot M. The Effects of Tryptophan on Everyday Interpersonal Encounters and Social Cognitions in Individuals with a Family History of Depression. Int J Neuropsychopharmacol. 2015; 18:1-8.

5. Rajkumar RP, Bharadwaj B. Dhat syndrome: evidence for a depressive spectrum subtype. Asian J Psychiatr. 2014; 9:57-60.

6. Maradiegue AH, Lyon DE, Meyers MF. Using family history and health risk behaviors to determine predictors of depressive symptoms in Central American immigrant mothers. Nurs Health Sci. 2013; 15:220-228.

7. Berton O, Nestler EJ. New approaches to antidepressant drug discovery: beyond monoamines. Nat Rev Neurosci. 2006; 7:137-151.

8. Anand A, Li Y, Wang Y, Wu J, Gao S, Bukhari L, Mathews VP, Kalnin A, Lowe MJ. Activity and connectivity of brain mood regulating circuit in depression: a functional magnetic resonance study. Biol Psychiatry. 2005; 57:1079-1088.

9. Hirschfeld R, Weissman M. Risk factors for major depression and bipolar disorder. In: Davis KL, Charney D, Coyle JT, Nemeroff C, Editors. Neuropsychopharmacology: the fifth generation of progress. Philadelphia, Penn: Lippincott, Williams, \& Wilkins. 2002; pp. 1017-26.

10. Li W, Chen Z, Wu M, Zhu H, Gu L, Zhao Y, Kuang W, Bi F, Kemp GJ, Gong Q. Characterization of brain blood flow 
and the amplitude of low-frequency fluctuations in major depressive disorder: A multimodal meta-analysis. J Affect Disord. 2017; 210:303-311.

11. Kong XM, Xu SX, Sun Y, Wang KY, Wang C, Zhang J, Xia JX, Zhang L, Tan BJ, Xie XH. Electroconvulsive therapy changes the regional resting state function measured by regional homogeneity ( $\mathrm{ReHo}$ ) and amplitude of low frequency fluctuations (ALFF) in elderly major depressive disorder patients: An exploratory study. Psychiatry Res. 2017; 264:13-21.

12. Zhou M, Hu X, Lu L, Zhang L, Chen L, Gong Q, Huang X. Intrinsic cerebral activity at resting state in adults with major depressive disorder: A meta-analysis. Prog Neuropsychopharmacol Biol Psychiatry. 2017; 75:157-164.

13. Zou K, Gao Q, Long Z, Xu F, Sun X, Chen H, Sun X. Abnormal functional connectivity density in first-episode, drug-naive adult patients with major depressive disorder. J Affect Disord. 2016; 194:153-158.

14. Drevets WC. Neuroimaging studies of mood disorders. Biol Psychiatry. 2000; 48:813-829.

15. Guo WB, Sun XL, Liu L, Xu Q, Wu RR, Liu ZN, Tan CL, Chen HF, Zhao JP. Disrupted regional homogeneity in treatment-resistant depression: a resting-state fMRI study. Prog Neuropsychopharmacol Biol Psychiatry. 2011; 35:1297-302.

16. Guo WB, Liu F, Xue ZM, Yu Y, Ma CQ, Tan CL, Sun XL, Chen JD, Liu ZN, Xiao CQ, Chen HF, Zhao JP. Abnormal neural activities in first-episode, treatment-naïve, shortillness-duration, and treatment-response patients with major depressive disorder: a resting-state fMRI study. J Affect Disord. 2011; 135:326-331.

17. Liu F, Guo W, Liu L, Long Z, Ma C, Xue Z, Wang Y, Li J, Hu M, Zhang J, Du H, Zeng L, Liu Z, et al. Abnormal amplitude low-frequency oscillations in medication-naive, first-episode patients with major depressive disorder: a resting-state fMRI study. J Affect Disord. 2013; 146:401-406.

18. Guo WB, Liu F, Xue ZM, Xu XJ, Wu RR, Ma CQ, Wooderson SC, Tan CL, Sun XL, Chen JD, Liu ZN, Xiao CQ, Chen HF, et al. Alterations of the amplitude of low-frequency fluctuations in treatment-resistant and treatment-response depression: a resting-state fMRI study. Prog Neuropsychopharmacol Biol Psychiatry. 2012; 37:153-160.

19. Guo W, Liu F, Yu M, Zhang J, Zhang Z, Liu J, Xiao C, Zhao J. Decreased regional activity and network homogeneity of the fronto-limbic network at rest in drugnaive major depressive disorder. Aust N Z J Psychiatry. 2015; 49:550-556.

20. Zhuo C, Zhu J, Wang C, Qu H, Ma X, Qin W. Different spatial patterns of brain atrophy and global functional connectivity impairments in major depressive disorder. Brain Imaging Behav. 2016; [Epub ahead of print].

21. Järnum H, Eskildsen SF, Steffensen EG, LundbyeChristensen S, Simonsen CW, Thomsen IS, Fründ ET, Théberge J, Larsson EM. Longitudinal MRI study of cortical thickness, perfusion, and metabolite levels in major depressive disorder. Acta Psychiatr Scand. 2011; 124:435-446.

22. Ota M, Noda T, Sato N, Hattori K, Teraishi T, Hori H, Nagashima A, Shimoji K, Higuchi T, Kunugi H. Characteristic distributions of regional cerebral blood flow changes in major depressive disorder patients: A pseudocontinuous arterial spin abeling (pCASL) study. J Affect Disord. 2014; 165:59-63.

23. Vasic N, Wolf ND, Grön G, Sosic-Vasic Z, Connemann BJ, Sambataro F, von Strombeck A, Lang D, Otte S, Dudek M, Wolf RC. Baseline brain perfusion and brain structure in patients with major depression: a multimodal magnetic resonance imaging study. J Psychiatry Neurosci. 2015; 40:412-421.

24. Li W, Chen Z, Wu M, Zhu H, Gu L, Zhao Y, Kuang W, Bi F, Kemp GJ, Gong Q. Characterization of brain blood flow and the amplitude of low-frequency fluctuations in major depressive disorder: A multimodal meta-analysis. J Affect Disord. 2017; 210:303-311.

25. Lindner T, Helle M, Jansen O. A Short Introduction to Arterial Spin Labeling and its Application to Flow Territory Mapping. Clin Neuroradiol. 2015; 2:211-218.

26. Hartkamp NS, Petersen ET, De Vis JB, Bokkers RP, Hendrikse J. Mapping of cerebral perfusion territories using territorial arterial spin labeling: techniques and clinical application. NMR Biomed. 2013; 26:901-912.

27. Guo W, Liu F, Liu J, Yu M, Zhang Z, Liu G, Xiao C, Zhao J. Increased cerebellar-default-mode-network connectivity in drug-naive major depressive disorder at rest. Medicine (Baltimore). 2015; 94:e560.

28. Alexopoulos GS, Kiosses DN, Heo M, Murphy CF, Shanmugham B, Gunning-Dixon F. Executive dysfunction and the course of geriatric depression. Biol Psychiatry. 58:204-210.

29. Davies J, Lloyd KR, Jones IK, Barnes A, Pilowsky LS. Changes in regional cerebral blood flow with venlafaxine in the treatment of major depression. Am J Psychiatry. 2003; 160:374-376.

30. Galynker II, Cai J, Ongseng F, Finestone H, Dutta E, Serseni D. Hypofrontality and negative symptoms in major depressive disorder. J Nucl Med. 1998; 39:608-12.

31. Wang Y, Zhang H, Tang S, Liu X, O'Neil A, Turner A, Chai F, Chen F, Berk M. Assessing regional cerebral blood flow in depression using 320-slice computed tomography. PLoS One. 2014; 9:e107735.

32. Li W, Chen Z, Wu M, Zhu H, Gu L, Zhao Y, Kuang W, Bi F, Kemp GJ, Gong Q. Characterization of brain blood flow and the amplitude of low-frequency fluctuations in major depressive disorder: A multimodal meta-analysis. J Affect Disord. 2017; 210:303-311.

33. Kaichi Y, Okada G, Takamura M, Toki S, Akiyama Y, Higaki T, Matsubara Y, Okamoto Y, Yamawaki S, Awai K. Changes in the regional cerebral blood flow detected by 
arterial spin labeling after 6-week escitalopram treatment for major depressive disorder. J Affect Disord. 2016; 194:135-143.

34. Guo W, Liu F, Xiao C, Zhang Z, Liu J, Yu M, Zhang J, Zhao J. Decreased insular connectivity in drug-naive major depressive disorder at rest. J Affect Disord. 2015; 179:31-37.

35. Murphy K, Birn RM, Bandettini PA. Resting-state fMRI confounds and cleanup. Neuroimage. 2013; 80:349-359.

36. Kolbitsch C, Lorenz IH, Hörmann C, Schocke M, Kremser C, Zschiegner F, Felber S, Benzer A. The impact of increased mean airway pressure on contrast-enhanced MRI measurement of regional cerebral blood flow ( $\mathrm{rCBF}$ ), regional cerebral blood volume $(\mathrm{rCBV})$, regional mean transit time (rMTT), and regional cerebrovascular resistance (rCVR) in human volunteers. Hum Brain Mapp. 2000; 11:214-222.
37. Zelaya FO, Zois E, Muller-Pollard C, Lythgoe DJ, Lee S, Andrews C, Smart T, Conrod P, Vennart W, Williams SC, Mehta MA, Reed LJ. The response to rapid infusion of fentanyl in the human brain measured using pulsed arterial spin labelling. MAGMA. 2012; 25:163-175.

38. Eklund A, Nichols TE, Knutsson H. Cluster failure: Why fMRI inferences for spatial extent have inated false-positive rates. PNAS 2016; 113:7900-7905.

39. Hamilton M. A rating scale for depression. J Neurol Neurosurg Psychiatry. 1960; 23:56-62. 